

\section{Decompressive craniectomy in the emergency setting: A historical review, summary of published evidence and review of implications for pre-hospital emergency care}

Hannah Kirby, first-year paramedicine graduate ${ }^{1}$; Jacki Burchell, first-year paramedicine graduate ${ }^{1}$; Jack Taylor, firstyear paramedicine graduate ${ }^{1}$

Affiliation:

${ }^{1}$ Department of Paramedicine, Victoria University, Melbourne, Victoria, Australia

\section{Abstract}

\section{Introduction}

Decompressive craniectomy is a controversial treatment for increased intracranial pressure resulting from traumatic head injuries. The technique has been around for approximately 5000 years but only now are researchers beginning to unlock its true potential. This article aims to summarise history, review current knowledge and identify the implications of pre-hospital emergency care.

\section{Methods}

An electronic search was conducted using the databases MEDLINE (via EBSCOHost), BioMed Central and Cochrane Database of Systematic Reviews.

\section{Results}

One hundred and thirty-one articles were identified. Articles excluded from analysis were those unavailable as full text, not available in English and review articles. Twenty articles were included in the analysis.

\section{Conclusion}

Decompressive craniectomy is a comparable treatment to alternative techniques. Until recently mortality rates have been misinterpreted and unfairly reported due to the severity of brain injuries patients had already suffered. Timing of decompressive craniectomy is a crucial factor of patient outcome, and it is of popular belief that the best chance of optimal recovery relies on a reduced injury to treatment time. Introducing decompressive craniectomy into the pre-hospital setting may see an increase in favourable outcomes and has the potential to save the health care system millions of dollars in associated costs for patients with traumatic brain injury.

Keywords:

decompressive craniectomy, craniotomy, intracranial hypertension, emergency neurosurgery 


\section{Introduction}

Traumatic brain injury (TBI) is a leading cause of disability and death, with the highest incidence occurring in young males. Raised intracranial pressure (ICP) caused from the $\mathrm{TBI}$ is responsible for a large majority of these deaths and disabilities $(1,2)$. The most definitive form of current treatment for increased ICP is decompressive craniectomy (DC). The technique of DC involves a scalp incision, surgically removing segments of bone from either one or both sides of the skull and opening of the dura mater. The aim of DC is to prevent brain compression from increased intracranial fluid and therefore preventing cerebral hypoxia (2-5). The swelling that occurs from a head injury results in increased cerebral fluid volume, the increased volume raises ICP which causes cerebral blood flow to decrease and subsequently causes a decrease in cerebral perfusion. The decrease of cerebral perfusion can lead to hypoxic brain injuries and magnify injuries from the primary insult. Furthermore, the increasing swelling can lead to transtentorial herniation, which can quickly lead to death without rapid treatment (6). The technique of $\mathrm{DC}$ has a long history, seen as early as $3000 \mathrm{BC}$, and has been seen as a controversial treatment since the 1960s (2,7). However, neurosurgeons are beginning to appreciate the potential of $D C$ and as a result the procedure has been steadily gaining popularity, although debate continues over the correct timing, indications and techniques (2). Decompressive craniectomy can be used to treat raised ICP in trauma, intracranial haemorrhage, hydrocephalus, cerebral abscesses, cerebral infarctions and other disorders and diseases $(8,9)$.

This review will touch on other causes but will focus mainly on trauma related cases. This review aims to summarise historical data and identify current knowledge based on relevant peerreviewed literature, while recognising the impact paramedics have on outcomes of patients with TBI.

\section{Methods}

An electronic search was conducted using the databases: MEDLINE (via EBSCOHost), BioMed Central and Cochrane Database of Systematic Reviews. The following keywords were included in the search: 'decompressive craniectomy', 'craniotomy', 'intracranial hypertension' and 'emergency neurosurgery'. Articles that were excluded from analysis were those unavailable in full text, unavailable in English, not peerreviewed and review articles.

\section{Results}

One hundred and thirty-one articles were identified, and from these 20 articles were identified as suitable for inclusion in the analysis. Details for the inclusion process are shown in Figure 1.

\section{Records identified by electronic database search \\ $(\mathrm{n}=131)$}

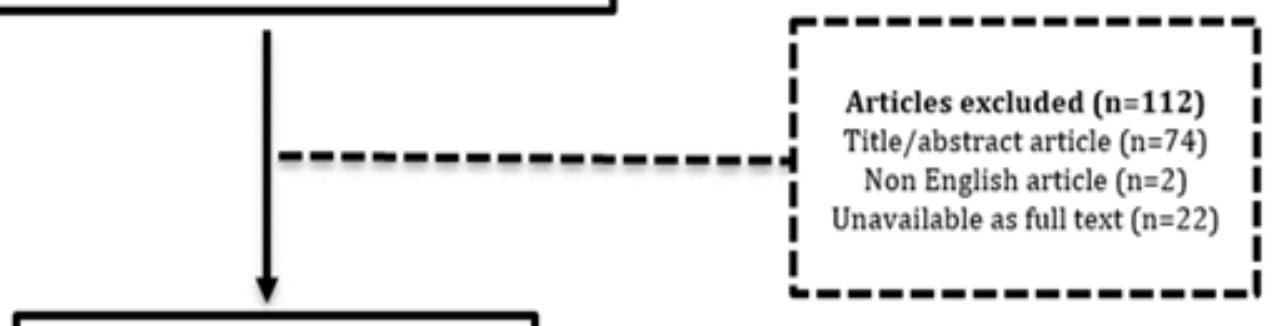

Total articles included in analysis

$(\mathbf{n}=\mathbf{2 0})$

Figure 1. Search results 


\section{Discussion}

\section{History}

Evidence of trepanation (surgically removing a section of skull) is seen as early as $3000 \mathrm{BC}$. It is believed that the origin of the technique is derived from both mythical and religious reasoning (7). Dr Paul Broca, a French neurosurgeon in the 1800s, discovered that some holes found in ancient skulls were caused by a methodical hand and were not trauma related. He speculated that the procedure was carried out in Neolithic times to create an exit point for demons that were causing convulsions. Furthermore, the Neolithic people believed that the bone fragment that was removed held great medical and spiritual importance and was worn as jewellery or made into pills, often sold in pharmacies as a preventive measure for convulsions. Broca became fascinated with the technique and began to experiment on cadavers and live dogs and through his studies he proved a craniectomy could be done on living patients without causing death (7).

Throughout the 1900s, Broca's theory was widely accepted, although it wasn't until the late 1960s that the procedure was introduced as a legitimate surgical intervention to treat increased intracranial hypertension (2). Popularity for the procedure plummeted after the majority of outcomes were unfavourable, resulting in severe disability, persistent vegetative state or death. Studies conducted at that time suggested that not only is there no benefit to the procedure, but may even worsen patient outcomes and prolong suffering. The procedure was abandoned until the 1980s when contradictory studies suggested the technique could have a positive outcome and potentially be a lifesaving intervention (10). Today the treatment is still seen as controversial, but is becoming more widely accepted in major hospitals.

\section{Technique}

The procedure itself is quite simple. It can be done either bilaterally or unilaterally and with or without opening of the dura mater, these decisions are made based on the extent and location of the injury. Regions of the craniectomy are carefully chosen as an inadequate opening may cause venous infarction or oedema at the margins (11). The aim is to reduce intracranial pressure to below $20 \mathrm{mmHg}$ while maintaining cerebral perfusion pressure of above $60 \mathrm{mmHg}$ (10). After a cranial computed tomography (CT) scan, the patient is placed under general anaesthesia via endotracheal intubation and the procedure is commenced. A myocutaneous flap is created to gain access to the skull and a bone segment is carefully removed. Regions of the skull often removed include frontal, parietal, temporal and occipital squama. After the removal of the bone segment, the dura mater is either left intact or incised in a stellate pattern. The opening of the dura not only allows the surgeon more comprehensive examination, but also allows for irrigation and removal of clots and irreparable contused brain matter. An ICP monitor is often placed under the dura and secured. A synthetic dura material can be placed to allow for the swelling of the brain. The myocutaneous flap is then resecured with sutures and the bone segment is stored in damp sterile gauze at a temperature of minus 70 degrees Celsius for cranioplasty $3-6$ months following $D C(2,3,10,11)$.

\section{Indications and outcomes}

Decompressive craniectomy indications vary from hospital to hospital, although all are quite similar. Common indications include bilateral or unilateral brain swelling as shown on CT scans, initial Glasgow Coma Scale (GCS) score of less than 8 or decreasing neurological status, intracranial hypertension of more than $30 \mathrm{mmHg}$ for a period of 15 minutes or more, unresponsive dilated pupils and the failure to respond adequately to other medical interventions $(2,9)$. Kim et al. (9) conducted a study to determine patients' outcomes after undergoing DC. The study followed 28 patients who underwent DC due to TBI. A favourable outcome was reported in $57.1 \%$ of patients while $21.4 \%$ of patients died and $21.5 \%$ of patients were left with a severe disability or in a vegetative state. It was deemed a 'favourable outcome' if the patient was left with a moderate disability or recovered entirely (9). The authors of the study suggested that the high rate of unfavourable outcomes may be due to the increased period of time waiting for the GCS to drop below 8, allowing further damage to occur within this time. Deok-Ryeong et al. (2) agreed, suggesting the poor outcomes of DCs could be a direct result of a few of the indications. By waiting for the patient to decline to meet guidelines, experimenting with alternative treatment and waiting the 15 minutes with increased intracerebral hemorrhage (ICH), irreparable damage could be occurring resulting in the high statistics of unfavourable outcomes $(2,9)$. Deok-Ryeong et al. (2) have proposed that to combat this problem DC should be a first line of treatment, performed as soon as practically possible in order to achieve the best possible outcome.

A group of researchers in Jordan in 2010, Jamous et al. (1) challenged the indications and directed a study on DC patients with a GCS score of 3 with bilateral fixed dilated pupils. Decompressive craniectomies were performed within 2-6 hours post-injury and ICP was seen to decrease, however within 30 days all patients had died. Jamous et al. argued that $\mathrm{DC}$ in these patients did not only prove unsuccessful, but prolonged the suffering of the patients and affected families. The authors of the study decided the $100 \%$ mortality rate was most likely due to the level of brain injury and these patients likely had no chance of survival regardless of treatment (1). These results should lead to further discussion regarding the exclusion of patients with a GCS score of 3 . 
Research has been directly responsible for improving patient outcomes. Research by Deok-Ryeong et al. (2) found that consistently monitoring ICP post-DC significantly improves patient outcome. Their study saw 78 patients with severe traumatic brain injury with a GCS of 9 or less split into two groups: a control group (53 patients) and a study group (25 patients). The study group had a subdural ICP monitor inserted under the dura matter and were given constant ICP monitoring for two weeks post-DC. The mortality rate for the control group was $50.9 \%$, while the group with ICP monitoring had a mortality rate of only $24.0 \%$ (2). This shows a significant improvement of outcomes and should encourage neurosurgeons to place an ICP monitor in all DC patients.

More research needs to be conducted to properly understand the outcomes of DC and how the indications affect success. Two randomised controlled trials (RCT) are currently underway: one in Europe (RescuelCP) and the other in Australia (DECRA), both are yet to publish any findings (12).

\section{Complications}

Honeybul et al. (10) recently completed a study on surgical complications of DC. Within the report Honeybul et al. states that complications arising from DC specific to head trauma patients were common, while Yang et al. (5) reported conflicting findings, stating the procedure has a 'low incidence of complications'. Honeybul et al. explores the complications in depth and their study included 270 patients who underwent both unilateral and bilateral decompression. The complications found attributable to DC were cortical herniation of cortical surface $(33.75 \%)$, injury of herniated cortex $(5.2 \%)$, seizures (18.5\%), cerebrospinal fluid (CSF) hydrodynamic disturbances including hydrocephalus (14.4\%) and subdural effusion (53.8\%). The complication incident rates concur with the conclusion of complications being common. Another complication Honeybul et al. (10) identified but did not include in the statistics was the death of patients caused by injury to the unprotected brain, of which two patients died from this cause.

\section{Cortical herniation}

Cortical herniation is the swelling of the brain, this becomes a concern when the herniated area is so severe that it protrudes from the confinement of the skull. The herniated area can become damaged from the margins of the skull or become injured from an external force; for these reasons patients who develop cortical herniation are at a higher risk of an unfavourable outcome (10).

\section{Hydrocephalus}

Hydrocephalus is the enlargement of the cerebral ventricles. The pathophysiology is poorly understood, however some researchers speculate it results from placing the medial margin of the craniectomy in close proximity of the midline. This condition can be difficult to differentiate from ventriculomegaly, however hydrocephalus usually shows signs of clinical improvement after the placement of a shunt (10).

\section{Subdural effusion}

Subdural effusion is the accumulation of fluid between the dura and subarachnoid space and commonly occurs within weeks of DC. Most subdural effusions resolve spontaneously or can be treated conservatively; surgical intervention is only considered when symptoms manifest. The more severe the head trauma, the more likely a patient is to develop this complication $(10,13)$. Subdural effusion normally presents unilaterally, however Yang et al. (5) found bilateral subdural effusion to occur in $6.5 \%$ of patients. Though rare, this is a more complicated manifestation that brings higher risk of a poor recovery (5).

\section{Paediatrics}

Paediatric patients have the potential for ICP to raise more dramatically and rapidly than adults and researchers are in agreeance that $\mathrm{DC}$ should be considered more quickly in children $(14,15)$. Fagaji et al. (4) studied a group of five paediatric patients who underwent $D C$ at the Red Cross Children's Hospital in Capetown, 2003. Patients ranged between the ages of five and 12, the group consisted of two females and three males. Surgeons responded quickly once symptoms of raised ICP appeared, with early and aggressive treatment given, $100 \%$ of patients resulted in a favourable outcome. While these results sound conclusive a limitation of the study is the small number of involved participants. It may be possible that children react more favourably to $D C$ than adults, however an RCT should be carried out to challenge this conclusion. Josan and Sgourus (16) compared paediatric patients who received $D C$ to those who received non-operative treatment. The DC group had a better outcome and concluded $\mathrm{DC}$ offers the fastest relief of $\mathrm{ICH}$ and is a safe procedure with a low rate of complications if performed quickly (16).

\section{Decompressive craniectomy versus craniotomy}

Decompressive craniectomy has been rigorously compared to craniotomy $(\mathrm{CR})$ in many studies. A craniotomy is similar to a DC, however the bone segment is replaced once the surgeon has finished treating the injured site. The clear advantage DC has over CR is the more effective control of ICP, however there is no guideline that states which of the two techniques should be performed, with surgeons currently using their prerogative (17). In 2012, Li et al. (17) completed a study in the United Kingdom evaluating the recovery of 91 patients who underwent either DC (51 patients) or CR (40 patients). Of the DC group, $67 \%$ of patients were male with a mean age of 45 years; the $\mathrm{CR}$ group had $50 \%$ males with a mean age of 59 years. At 6 months, patients were assessed and then deemed to have either a favourable (moderate disability or good recovery) or unfavourable (death, persistent vegetative state or severe disability) outcome. Patients in the DC group had $42 \%$ favourable outcomes ( $58 \%$ unfavourable) compared to 
Kirby: Decompressive craniectomy in the emergency setting

Australasian Journal of Paramedicine: 2017;14(1)

$45 \%$ in the CR group (55\% unfavourable). The overall results were not significant and the authors concluded that the rate of unfavourable outcomes in DC was high as patients with more severe TBls were in the DC group; once injury severity was accounted for the outcomes are quite similar (17).

In the United States, a similar study was conducted by Soukiasian et al. (6), the authors accessed database information, which included patients who underwent DC or CR from 1995 to 2001, the search resulted in 120 patients eligible for analysis. The DC group consisted of 24 patients, who had a mean age of 40 with a standard deviation (SD) of 3.9 and was comprised of $83 \%$ males. The CR group consisted of 96 patients, a similar mean age of 42 years with a SD of 2.2, $81 \%$ of which were male. The mortality rate for the DC group was $37.5 \%$ versus $18.8 \%$ for the CR group, the survival rate for the DC group was $52.8 \%$ versus $79.2 \%$ for the CR group. These rates concluded no significance between the groups in terms of survival $(p=0.08)(6)$. These rates conclude $D C$ is beneficial for patients with TBI and instil the importance of a future RCT. It is important to note that patients with the DC group had suffered more severe brain trauma than those in the CR group, which prevents this study from being definitive, as groups cannot be fairly compared. A further limitation of this study is the use of the database information; authors were not directly involved in the patient's recovery, resulting in a potential loss of vital information. Furthermore, the majority of these patients were males aged in their 40s, therefore the results could not be generalised.

\section{Pre-hospital implications}

Traumatic brain injuries are responsible for a large number of deaths and disabilities annually (2). A recent study conducted by Gershon Spitz et al. (20) found a significant financial effect on the health system amounting to a lifelong cost of $\$ 2.5$ million per patient suffering from a moderate TBI and $\$ 4.8$ million for those suffering a severe TBI.

Paramedics are usually the first to treat these patients, therefore it is imperative they are acutely aware of the warning signs associated with increased ICP, and understand the relevant guidelines as well as the importance of rapid transport to definitive care. At present no study or trial has been completed involving DC in the pre-hospital setting. Given the widely accepted conclusion that early DC results in lower mortality and morbidity rates and high associated health care costs, the natural progression is to suggest the implementation of a simplified version of DC into the prehospital setting $(4,6,14,15,18)$. Intensive care paramedics already have extensive training coupled with impressive knowledge. Burr holes (drilling holes into the skull to relieve ICP) are recognised as a simplified and justified alternative for DC in underequipped hospitals (19), with further research and collaboration with experienced neurosurgeons it is possible burr holes may earn a place in the pre-hospital setting in the future.

It is the authors' belief that intensive care paramedics, equipped with extra training of the burr hole procedure, could potentially decrease the high disability and mortality rate following severe TBI: a RCT and further research would be worthwhile and is strongly recommended. Depending on the results of the RCT, factors such as technique, distance to hospital, injury to treatment time, patient age and the cost of introducing the procedure would all have to be addressed. Using the multiple burr hole technique over the single burr hole technique may prove favourable due to lack of radiographic equipment available preventing operators determining the location of the increased cranial pressure, this also decreases the amount of surgical equipment that needs to be carried in ambulances, and decreases the level of training required. The cost of introducing this procedure into the pre-hospital setting is unknown at this time, however introduction of pre-hospital DC has the potential to save millions of dollars on associated TBI health care costs (20). Training those most likely to utilise the procedure such as rural intensive care paramedics and air ambulance paramedics (due to the time and distance to a major trauma hospital), and providing only rural ambulances and helicopters with equipment will help keep the introduction of $D C$ cost effective.

\section{Conclusion}

Severe head trauma is a leading cause of death and no treatment is available until arriving at definitive care. The high mortality and morbidity rates seen associated with DC are more likely to be associated with the injuries suffered before undergoing treatment. The misinterpretation of the mortality rates has caused a considerable amount of controversy relating to $\mathrm{DC}$, but as more research comes to light neurosurgeons are beginning to consider $\mathrm{DC}$ an acceptable treatment with great potential. Decompressive craniectomies are seen to have similar outcomes to craniotomies, although further research is needed to conclude the most beneficial timing and technique for the procedure. Many researchers believe favourable outcomes depend on the prompt delivery of $\mathrm{DC}$, which may lead to simplified craniectomies being performed by rural intensive care paramedics. Not only does this have the potential to increase favourable outcomes from TBls, but also save the health care system what could amount to billions of dollars.

\section{Conflict of interest}

The authors declare they have no competing interests. Each author of this paper has completed the ICMJE conflict of interest statement. 


\section{References}

1. Jamous M, Barbarawi M, Samrah S, Khabaz M, Al-Jarrah M, Dauod S. Emergency decompressive craniectomy for trauma patients with Glasgow Coma Scale of 3 and bilateral fixed dilated pupils. Eur J Trauma Emerg Surg 2010;36:4659.

2. Kim D, Yang S, Sung J, Lee S, Son B. Significance of intracranial pressure monitoring after early decompressive craniectomy in patients with severe traumatic brain injury. $J$ Korean Neurosurg Soc 2014;55:26.

3. Fletcher T, Kolias A, Hutchinson P, Sutcliffe M. Development of a finite element model of decompressive craniectomy. PLoS ONE 2014;9:e102131.

4. Figaji A, Fieggen A, Peter J. Early decompressive craniotomy in children with severe traumatic brain injury. Child's Nervous System. 2003;19(9):666-673.

5. Yang X, Wen L, Li G, Zhan R, Ma L, Liu W. Contralateral subdural effusion secondary to decompressive craniectomy performed in patients with severe traumatic brain injury: incidence, clinical presentations, treatment and outcome. Med Princ Pract 2009;18:16-20.

6. Soukiasian H, Hui T, Avital I, et al. Decompressive craniectomy in trauma patients with severe brain injury. Am Surg 2002;68:1066-71.

7. Clower W, Finger S. Discovering trepanation: the contribution of Paul Broca. Neurosurgery 2001;49:1417-26.

8. Treacy P, Reilly P, Brophy B. Emergency neurosurgery by general surgeons at a remote major hospital. ANZ J Surg 2005;75:852-7.

9. Kim K, Park J, Kang S, et al. Comparison of the effect of decompressive craniectomy on different neurosurgical diseases. Acta Neurochir (Wien) 2008;151:21-30.

10. Honeybul S, Ho K. Decompressive craniectomy for severe traumatic brain injury: The relationship between surgical complications and the prediction of an unfavourable outcome. Injury 2014;45:1332-9.

11. El Hindy N, Stein K, Hagel V, Dammann P, Sure U, Mueller
0 . The role of decompressive craniectomy in children with severe traumatic brain injury. Eur J Trauma Emerg Surg 2013:40:481-7.

12. Eberle B, Schnuriger B, Inaba K, Peter Gruen J, Demetriades D, Belzberg $H$. Decompressive craniectomy: Surgical control of traumatic intracranial hypertension may improve outcome. Injury 2010;41:894-8.

13. Wang H, Lu K, Liang C, Tsai Y, Wang K, Liliang P. Contralateral subdural effusion related to decompressive craniectomy performed in patients with severe traumatic brain injury. ibid. 2012;43:594-7.

14. Csokay A, Emelifeonwu J, Fugedi L, Valalik I, Lang J. The importance of very early decompressive craniectomy as a prevention to avoid the sudden increase of intracranial pressure in children with severe traumatic brain swelling (retrospective case series). Childs Nerv Syst 2011;28:441-4.

15. Von Lehe M, Kim H, Schramm J, Simon M. A comprehensive analysis of early outcomes and complication rates after 769 craniotomies in paediatric patients. Childs Nerv Syst 2012;29:781-90.

16. Josan V, Sgouros S. Early decompressive craniectomy may be effective in the treatment of refractory intracranial hypertension after traumatic brain injury. ibid. 2006;22:126874.

17. Li L, Kolias A, Guilfoyle M, et al. Outcome following evacuation of acute subdural haematomas: a comparison of craniotomy with decompressive craniectomy. Acta Neurochir 2012;154:1555-61.

18. Clark D, Kolias A, Corteen E, et al. Community consultation in emergency neurotrauma research: results from a preprotocol survey. ibid. 2013;155:1329-34.

19. Fatigba H, Allodé A, Savi de Tové K, Mensah E, Hodonou A, Padonou J. The exploratory burr hole: indication and results at one departmental hospital of Benin. ISRN Surg 2013;2013:1-4.

20. Spitz G, Mckenzie D, Attwood D, Ponsford J. Cost prediction following traumatic brain injury: model development and validation. J Neurol Neurosurg Psychiatry 2016;87:173-80. 